\title{
High harmonic generation in solids driven by sub-cycle mid-infrared pulses from laser filamentation
}

\author{
Fumitoshi Kumaki ${ }^{1,2}$, Hideto Shirai ${ }^{1}$, Yutaka Nomura $^{1}$, and Takao Fuji ${ }^{1, *}$ \\ ${ }^{1}$ Institute for Molecular Science, 38 Nishigonaka, Myodaiji, Okazaki 444-8585, Japan \\ ${ }^{2}$ The Graduate University for Advanced Studies (SOKENDAI), Myodaiji, Okazaki 444-8585, Japan
}

\begin{abstract}
Carrier-envelope phase (CEP) controlled subcycle mid-infrared pulses from two-color laser filamentation have been applied for high harmonic (HH) generation in a crystalline silicon membrane. The HH spectrum reaches the ultraviolet region $(<300 \mathrm{~nm})$, beyond the direct band gap of the silicon. The shape of the HH spectrum shows the strong dependency on the CEP of the input pulse. The complete waveform characterization of the sub-cycle driver pulse with frequency-resolved optical gating capable of CEP determination is the effective method for the studies of the sub-cycle dynamics.
\end{abstract}

\section{Introduction}

In the last few years, high harmonic generation (HHG) in solids has been actively researched in the field of ultrafast science and solid-state physics. HHG in solids would be more efficient since the atomic density is much higher than in gases. Therefore, HHG in solids is expected to become a key technology to realize compact solid-state attosecond pulse generator and $\mathrm{PHz}$ electronics.

One of the most straightforward approaches to investigate the single-cycle ultrafast phenomena like HHG is the experimental study with well-characterized single-cycle or sub-cycle pulses [1]. It enables us to observe the phenomena driven by single-cycle fields. In the MIR experiment, the waveform dependence, namely carrier-envelope-phase (CEP) dependence, of the high-harmonic $(\mathrm{HH})$ spectra has not been studied. In this contribution, we report the HHG in the silicon membrane driven by CEP-controlled sub-cycle MIR pulses generated through filamentation.

\section{Experiment}

The light source was based on a Ti:sapphire multipass amplifier $(800 \mathrm{~nm}, 30 \mathrm{fs}, 0.85 \mathrm{~mJ}$ at $1 \mathrm{kHz}$, Femtopower compactPro, FEMTOLASERS). CEP stable sub-cycle MIR pulses ( $4 \mu \mathrm{m}, 8.5 \mathrm{fs}, 360 \mathrm{~nJ})$ were generated from two-color filamentation [2]. The intensity and electric field strength of the MIR pulse was estimated as about $0.5 \times 10^{12} \mathrm{~W} / \mathrm{cm}^{2}$ and $0.18 \mathrm{~V} / \AA$. The waveform of the MIR pulse was characterized with frequency-resolved optical gating capable of the CEP determination (FROG-CEP) [3]. The characterized MIR pulse was focused onto a crystalline (100) silicon membrane ( $\mathrm{Si}, t=200 \mathrm{~nm}$, Norcada). The spectrum of the $\mathrm{HH}$ generated from the back surface of the membrane was introduced into a spectrograph with an electron-multiplying CCD camera (SP-2358 and ProEM1600, Princeton Instruments).

\footnotetext{
*e-mail: fuji@ims.ac.jp
} 


\section{Result}

Figure 1(a) shows a power spectrum and spectral phase of the MIR pulse measured with FROG-CEP. The power spectrum is broader than three octaves. The spectral phase has some nonlinear term, which means that the MIR pulses are slightly chirped. Figure 1(b) shows the characterized waveforms of the subcycle MIR pulses for the phases of $-0.10 \pi$, and $-0.77 \pi$ at $4 \mu \mathrm{m}$. Figure 2 shows the $\mathrm{HH}$ spectra driven by the MIR pulses shown in Fig. 1(b). The HH spectrum reaches the ultraviolet region $(<300 \mathrm{~nm})$. The shape of the HH spectrum
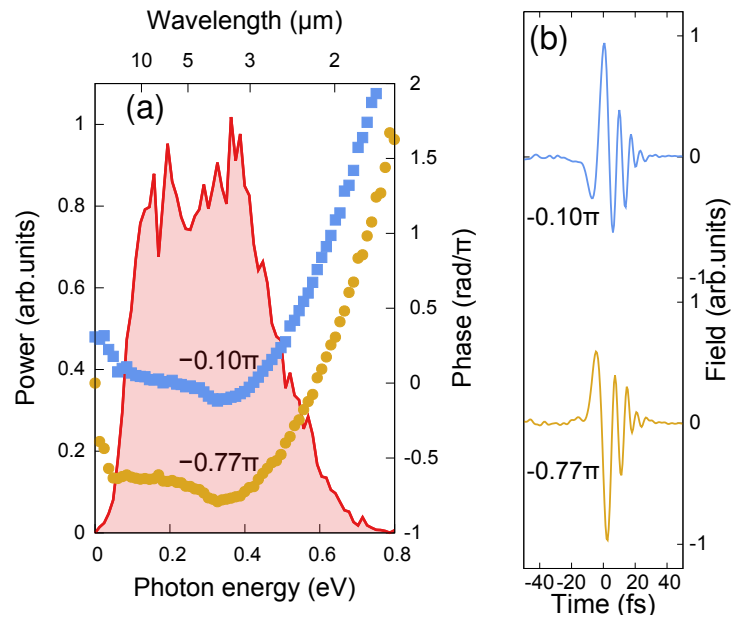

Figure 1. (a) Power spectrum and absolute spectral phases. (b) Retrieved waveforms of the MIR pulses. is very complex. There is no clear structure such as plateau or cutoff in the spectrum, even the orders of the harmonics is unclear.

\section{CEP dependence of the HH spectrum}

Figure 3(a) shows the CEP dependence of the $\mathrm{HH}$ spectrum recorded continuously while CEP scanning from $-\pi$ to $\pi$. Three features can be seen in this figure. First is that the HH spectrum shifts to higher photon energy region as the CEP of the MIR pulse increases. Second is that the same spectrum appears at every $\pi$ phase shift. Third is that the CEP dependence of the $\mathrm{HH}$ spectrum discontinuously changes at around $3.1 \mathrm{eV}$, which corresponds to the direct band gap energy of $\mathrm{Si}$. We believe that this change is due to the increase in the imaginary part of the refractive index at around the direct band gap.

To investigate the complex structure and CEP dependence of the $\mathrm{HH}$ spectrum, we numerically simulated the CEP dependence of the HH spectrum based on the optical Bloch equations generalized to the case of a two-band semiconductor [4]. In this numerical simulation, we used the waveform of the sub-cycle MIR pulse measured with FROG-CEP as a driving field.

Figure 3(b) shows the HH spectrum CEP dependence obtained by the simulation. As is the case with experimental results in Fig. 3(a), the structure of the HH spectrum shifts to higher photon energy region by increasing of the CEP of the driving pulses and, the same spectrum appears at every $\pi$ phase shift. There is also the discontinuous change of the CEP 
dependence of the $\mathrm{HH}$ spectrum at around $3.1 \mathrm{eV}$. In this simulation, the main features of the experimental result have been reproduced qualitatively.

Next, to clarify the effect of subcycle driving pulse, we simulated the CEP dependence of the $\mathrm{HH}$ spectrum with the transform-limited pulses, obtained by assuming a flat phase with the same spectrum measured with FROGCEP. Figure 3(c) is the CEP dependence of the $\mathrm{HH}$ spectrum simulated by using the transform-limited pulse. In contrast to the simulation result obtained by using the waveform measured in the experiment, the spectrum does not shift to higher photon energy region by changing the CEP. This result indicates that the CEP dependence of the $\mathrm{HH}$ spectrum is very sensitive to the chirp of the driver pulse.

\section{Conclusion}

The sub-cycle MIR pulse generated through filamentation has enough intensity to induce nonlinear effects in solids. The HH spectrum in a silicon membrane driven by the sub-cycle pulse spread to the ultraviolet region $(<300 \mathrm{~nm})$. Since the spectrum of the sub-cycle pulse is broader than three octaves, many orders of the harmonics interfere with each

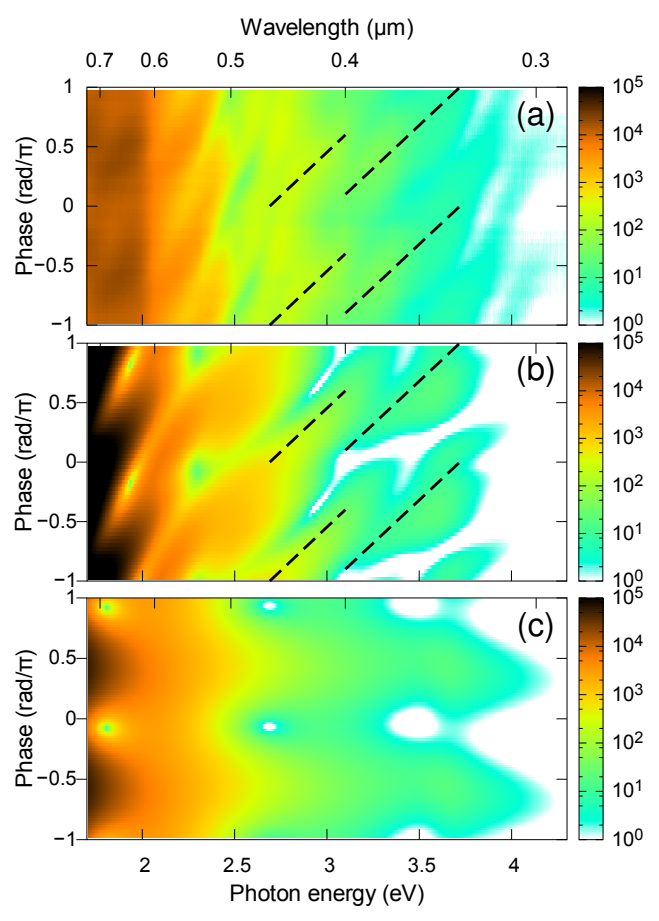

Figure 3. CEP dependence of the HH spectrum. (a) Experimental result. (b) Numerical simulation result obtained from optical Bloch equations. (c) Simulation result with the transform-limited pulses. other. The interference signal of the $\mathrm{HH}$ spectrum is very sensitive to the CEP of the input pulse. Therefore, FROG-CEP measurement which can determine the waveform of the pulse including the absolute CEP value is very useful to investigate field sensitive nonlinear phenomena in solid state [5].

\section{References}

[1] T. T. Luu, M. Garg, Y. Kruchinin, A. Moulet, M. T. Hassan, and E. Goulielmakis, Nature 521, 498 (2015).

[2] T. Fuji, Y. Nomura, and H. Shirai, IEEE J. Sel. Quantum Electron. 21, 8700612 (2015).

[3] Y. Nomura, H. Shirai, and T. Fuji, Nat. Commun. 4, 2820 (2013).

[4] C. F. Klingshirn, Semiconductor Optics (Graduate Texts in Physics, Springer, 2012) 813.

[5] H. Shirai, F. Kumaki, Y. Nomura, and T. Fuji, Opt. Lett. 43, 2094 (2018). 